\title{
The Application of Value Analysis in the Product Redesign
}

\author{
FEI HE ${ }^{1, a}$ \\ ${ }^{1}$ School of Art And Design ,Jingdezhen Ceramic Institute,Jiangxi,China \\ ah_fei1225@126.com
}

\begin{abstract}
Keywords: Product redesign; value analysis; product value
Abstract. The redesign of the exiting products and the value analysis are powerful tools for the enterprises to participate in the market competition. For consumers, they are more willing to purchase satisfying products at low prices, so in the process of product redesign, people will be more likely to buy them when the enterprises strive to improve their application value and emotional value. Product design can determine its value, in reverse terms, the use of value analysis in product design can also promote the improvement of products, and provide ways of product redesign. Based on full study of the product redesign and value analysis of the product, this essay concludes the complementary relationship between them, which will affect the result of competition between enterprises directly.
\end{abstract}

\section{Introduction}

In the 1980s and the 1990s, from the point of view of fast production and acting with discretion, most of the manufacturers in China cannot wait selling products after imitating or making slight modifications to foreign products, resulting in serious homogenization of similar products in the market and lake of innovation. Due to the short period of design and production, products are short of application value and emotional value, from which the drawbacks in the process of industrialization appear. In order to meet the needs of modern society people's pursuit of convenient and comfortable life, and satisfy people's spiritual and material demands, from the perspective of less economic waste, the improvement of the exiting products is a more economical and feasible method, at the same time, the method of improving and optimizing the existing products is the basic way for development and expansion of enterprises, which is also a practical and feasible path for the development of the majority of small and medium enterprises in China, in fact, the development of the world's most large enterprises also follow this way.

\section{Product value analysis}

As the saying goes: 'No good cheap goods, good goods are not cheap.' Consumers are in the hope of buying good products at reasonable prices, manufacturers are also hoping to produce inexpensive high-quality products to earn more profits while winning more consumers. Beautiful package, excellent performance, convenient operation and reasonable price are the factors which may encourage purchase. Value analysis is to explore the relationship between price and function, for a certain product, price and value is inseparable, value analysis is to study products value from economic and technical point of view, and explore ways to enhance the product value by studying the product function and cost.

Generation and development of value analysis. Value analysis (VA, i.e. value analysis), also known as value engineering (VE, i.e. value engineer), is a new management technology, which provides high value products through the rationalization of the function and the cost of products, and improves the technical and economic benefits of the enterprise. Value analysis started from the United States in 1940s, due to the material consumption increase during wartime, also in order to ensure the performance of weapons and timely delivery, which resulted in abuse of limited resources and sharpening of the contradiction between the material shortage and the great consumption. American General Electric Corp was in urgent need of asbestos board at that time, design engineer Miles (L.D.Miles) put forward an idea that to replace asbestos board with non burning paper which features 
low price and adequate supply. Thus, Miles was inspired to study people's purchase behavior from the mentioned raw material substitutes event, he found out that, when purchasing products, people were not expecting the products themselves, but the a certain applied function it carried, when met difficulties in purchasing a certain kind of raw materials and product, people tend to choose more inexpensive ones or the ones with similar functions. Miles's research results can be summed up as follows: 1. When purchasing products, users are not buying products themselves, but the functions they carried. 2. When purchasing the functions that products carried, users expect less cost. 3. The concept of 'value analysis' can be proposed from the relationship between the function and the cost. 4 . Methods of improving product value can be achieved by studying the relationship between the function and the resources invested, and to improve the value of the product using the above methods, this is called value analysis.

Ways to improve product value. For products, the utility is the functions of their own, while resources invested can be regarded as cost for realization of these functions, so the value of the products can be expressed by the following formula:

The value of the product $=$ the product function / cost for realization of the function

If you use $\mathrm{F}$ to represent the function of the product, $\mathrm{V}$ to present the value of the product, $\mathrm{C}$ is expressed as the cost of the realization of the function. Then the expression form of the value of the products can be changed into: $\mathrm{V}=\mathrm{F} / \mathrm{C}$, it can be seen that, the value of the product is proportional to the function of the product, while the value of the product is inversely proportional to the cost of the realization of the function.

\section{Product redesign}

Product redesign is an industrial modeling design form based on the existing products, is a design activity closely related to creativity, creative thinking, invention spirit and technological innovation, and is a re-design process of exiting products. So the product redesign should be on the basis of investigation, analysis and understanding of existing products, with the aid of which, the 'disadvantages' and 'advantages' can be analyzed and judged objectively and comprehensively. To analyze the use environment and use conditions. In order to meet consumers' demands, many enterprises will launch many new products to the market every year, among which, the vast majority are the improved version of the original products, for many enterprises, this is not only a best way of less investment, quick returns, small risks, and less cost, but also an effective way for reducing products update cycle and rapid withdrawal of circulating funds. At the same time, the product redesign makes it more consistent with general principles of ergonomics in the premise of ensuring the product functions, therefore redesigned products are improved in terms of efficiency, safety, health and comfort properties, and humanity. After improvement, ways of production, consumption and recycling have become more transparent, each decision in the design process is fully considering the environmental benefits and reducing the damages to the environment, so the product redesign is also a systematic design of products and environment. Whether from the aspect of economic benefit, or the people-oriented design principle, even environmental protection, product redesign has advantages to a certain degree, however, the issues, such as the specific implementation methods, the process and the aspects to be improved, etc., are worth being studies carefully.

\section{Relationships between value analysis and the product redesign}

Value analysis provides product redesign with basic methods. From above, it can be seen that the increase of product value depends on the changes of relationships between the function and the cost of the functions. Based on the concept of value analysis, five methods of improving product value can be obtained;The first one: the product value can be greatly improved with the increase of the functions and decrease of the cost.The second: To increase the functions and the product value on the basis of unchanged cost.The third: To decrease the cost and increase the value with unchanged function.The forth: To increase the cost slightly and increase the function greatly, which results in the increased 
value.The fifth: To decrease the functions and decrease the cost significantly, therefore, the value will be increased.

From these five methods, it can be summed up that the aspects to be redesigned which are involve in the product redesign are as follows:

Improvement of internal structure:In the product value analysis, the most ideal way to improve the product value is the first one: that is to decrease the cost while increasing the function, resulting in greatly improved value. To use the first way in the product redesign, it can be obtained that the redesigned aspects, which are involved in the product redesign, contain the improvement of internal structure, for example: the development and application of electronic technology result in the the fact that electronic tube was replaced by the transistor, and then the transistor was replaced by the integrated block, which reflected that the cost was going lower and lower, while the function was going higher and higher, at the same time, the weight and internal structure of electronic products are changing with the use of the integrated block. In 2014, LG and Samsung were working on 18 inch flexible rollable OLED screen technology, with which the screen could be rolled as a scroll. This screen could be arbitrarily curved and not easy to break, at the same time, it made the cost of OLED screen lower through the control of the modes of production changes and the selection of raw materials. In today's society, due to the limitation of living space, many families will choose to purchase folding products, which will save space, as well as reduce transportation costs after being folded. Many families living in small-sized apartments will choose folding bathtub, a folding bathtub called 'Bathboard' has the common features of a bathtub, except which, it can be folded easily due to its soft and elastic foam rubber material and flexible drainage pipe design, also its weight is no more than $3 \mathrm{~kg}$ after being folded occupying a space of 12 centimeters from the wall. In addition to enhance the use functions, folding structure of products also save the transportation costs, and enhance their value intangibly. Such a product structure form is also the product redesign should be involved.

Changes in appearance :From the five ways for increasing value of the products, we can also draw the conclusion that product redesign can also be changes of product appearance, because to improve the function of the product not only refers to to improve the function of use, but also to improve the aesthetic function. To change the old forms of products, making the redesigned ones to show a distinct appearance or texture, which increases their aesthetic function, improves their value, at the same time, encourages consumption. In 2013, the famous French designer Philippe Starck designed a hard disk with a capacity of 4 TB and unique appearance, its source of inspiration came from the mysterious and magical power of the information technology, Philippe Starck combined angle shaped shell and metal materials together, to create an atmosphere of high tech, while making its appearance be different from other ones' in the market. Coincidentally, Dutch designer Marcel Wanders designed a series of lace-trim-patterned porcelain named 'DRESSED', which was inspired by the movie Black Swan, to re interpret decoration technique of classical tableware. White tableware with lace flower relief as basement, cup design discarded the smooth edge, but adopted flower-like rim, which looks as elegant and charming as ballet vests, at the same time, it depicted the same pattern on the back, the simple appearance showed a unique chilly feelings of ceramic. At the same time, the configuration of black and white colors also echoed the roles of black and white swans. Since then, Marcel Wanders had used this pattern and decorative techniques to a series of pots designs, and creates a generation of elegant kitchen utensils. And in addition to the way of enhancing aesthetic function by changing product texture effect, the appearance can be improved by changing the size of existing products, making it smaller, lighter and more convenient to carry while keeping the existing functions. Taiwan, China has produced an electronic watch, whose most distinctive feature is thin, with the thickness of only $0.8 \mathrm{~mm}$, even thinner than credit card. material of body and strap is stainless steel, and $0.5 \mathrm{~mm}$ thick flexible electronic ink display is attached on stainless steel substrates. It can be known that the way to improve the aesthetic function of the products (that is to change the appearance of the products) is also involved in the design improvement of products.

Changes of materials:From the perspective of value analysis, the use of new materials in the product will lead to the product function improvement and cost reduction, which is one of the ways to enhance 
the value of the products. So change of existing materials is also a redesigned aspect which should be involved in product redesign. In 2014, the anti pollen masks produced by ARAX Co.,LTD., was awarded the 2014 Good Design Award. The mask is made from soft and highly elastic polyurethane, which is different from active carbon fiber being used by general filter masks. Active carbon fiber will cause skin sensitivity if wore for a long time, but polyurethane material will not, at the same time, it possess a three-dimensional network structure which can capture pollen to the maximum degree. This mask can be reused, whose filterability of pollen is up to $99 \%$ after being washed for three times. This anti pollen mask design is to comply with the facial contour with excellent air permeability, which highly improved the situation that the filter performance and ventilation can not be achieved at one time. In addition to the use of new materials, under the circumstance of unchanged product functions, the use of protection material or the recycling of waste material makes effective use of residual value, thus lowering the costs, while enhancing the product value, it should also be involved in the product redesign. For example, the chair made of corrugated paper, goods manufactured with a variety of recycled materials, lamps made of wast hangers, paper boxes and gears.

Changes of manipulation:The five methods of increasing product value show that, convenient manipulation also improves product value intangibly, so the improvement of product manipulation should also be involved in the product redesign. Before 2007, the operation of mobile phones also need the help of various physical buttons, but at the beginning of 2007, the iPhone released by Apple used touch technology for the first time, then this technique is used by other handset makers. The use of touch technology had changed the operation of traditional mobile phones and promoted the development of smart phones. It can be seen that, the improved ideal product operation mode can be simple to present, and be accurately understood by the users, so as to achieve the purpose of improving the efficiency of the operation.

The mutual promotion relationship between the product redesign and the value analysis of the products. In addition to the aspects to be improved which has been provided for the product redesign by the mentioned value analysis, the product redesign also expends the application of value analysis, value analysis should deal with all kinds of value analysis and evaluations, but not just pay attention to the function value, besides, it should also analyze from the perspectives, such as product appearance, ergonomics and emotional factors, as well as costs and residual value from products transport to recovery process. It is important for both sides to learn from each others' strengths and advantages by combining value analysis and product redesign together, to make up the defects during the use of these methods, so as to make more popular design and more competitive products.

\section{Conclusion}

The purpose to use the product redesign and value analysis in the product design is to develop and produce more reasonable and popular products. To take the value analysis as a guidance in the product redesign, can provide the improvement of the exiting products. At the same time, in the practical application, the product redesign can be used to expend the scope of value analysis. It can be seen that the promotional relationship between the product redesign and the value analysis. To create a high-value product or function to replace the original scheme of low value in the product design through the product redesign and value analysis, is an effective method to improve the technical and economic benefits for enterprises.

\section{References}

[1] Jikun Liu:Product Value Analysis. Harbin: Heilongjiang Science and Technology Publishing House. ( 1997 )

[2] Dakang Xie:Thinking and Methods of Product Design. Beijing: Higher Education Press. ( 2005-6 